\title{
Effect of boric acid mixture as solid lubricant towards machining processes
}

\begin{abstract}
Milling is widely used metal removal process in manufacturing industry that involves generation of high cutting forces and temperature. Lubricants become important to reduce the cutting force and temperature for better machining processes and performances. Conventional cutting fluid has some limitations. The applications of conventional cutting fluid create some techno-environmental problems like environmental pollution, biological problems to operators and water pollution. Application of solid lubricant in milling has proved to be a feasible alternative to the conventional cutting fluids. The present work investigates the effect of boric acid as solid lubricant towards machining performances such as tool wear and surface roughness. The results indicate that boric acid can improve the cutting processes and performance compared to conventional cutting fluid.
\end{abstract}

Keyword: Boric acid; Cutting fluid; Lubricant; Machining process; Solid lubricant 